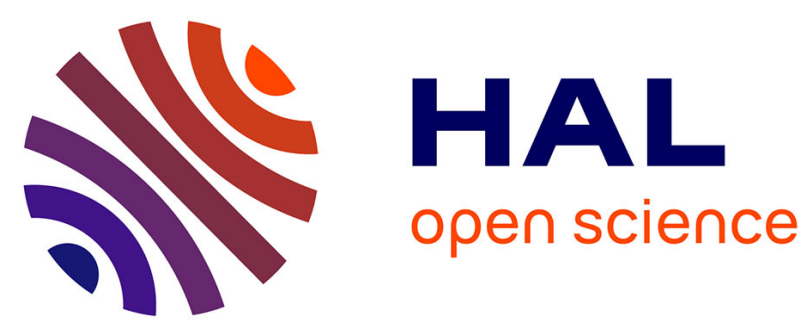

\title{
DENSITÉ DES NIVEAUX DE BASSE ÉNERGIE ET INSTABILITÉS DE SURFACE DANS LES NOYAUX LOURDS DE TRANSITION
}

W. de Wieclawik, J. Dionisio, R. Foucher, A. Hoglund, Ch. Vieu, W. Watzig

\section{- To cite this version:}

W. de Wieclawik, J. Dionisio, R. Foucher, A. Hoglund, Ch. Vieu, et al.. DENSITÉ DES NIVEAUX DE BASSE ÉNERGIE ET INSTABILITÉS DE SURFACE DANS LES NOYAUX LOURDS DE TRANSITION. Colloquium on Nuclear Surface, 1975, Dijon, France. pp.C5-83-C586, 10.1051/jphyscol:1975515 . jpa-00216370

\section{HAL Id: jpa-00216370 https://hal.science/jpa-00216370}

Submitted on 1 Jan 1975

HAL is a multi-disciplinary open access archive for the deposit and dissemination of scientific research documents, whether they are published or not. The documents may come from teaching and research institutions in France or abroad, or from public or private research centers.
L'archive ouverte pluridisciplinaire HAL, est destinée au dépôt et à la diffusion de documents scientifiques de niveau recherche, publiés ou non, émanant des établissements d'enseignement et de recherche français ou étrangers, des laboratoires publics ou privés. 


\title{
DENSITÉ DES NIVEAUX DE BASSE ENERGIE ET INSTABILITÉS DE SURFACE DANS LES NOYAUX LOURDS DE TRANSITION
}

\author{
W. DE WIEClAWIK $(*)$, J. S. DIONISIO $\left({ }^{* *}\right)$, R. FOUCHER $\left({ }^{*}\right)$, A. HOGLUND $\left({ }^{* * *}\right)$, \\ CH. VIEU $\left({ }^{* *}\right)$ et W. WATZIG $(* * * *)$
}

\begin{abstract}
Résumé. - Une analyse statistique des niveaux nucléaires de $\mathrm{Au}, \mathrm{Pt}, \mathrm{Hg}$ a été réalisée avec la méthode d'Ericson. Les distributions expérimentales du nombre de niveaux d'or impair sont comparées aux distributions théoriques correspondant aux modèles vibrationnels (Alaga, KisslingerSorensen) et rotationnels (Stephens, Meyer-ter-Vehn). Le modèle d'Alaga donne la description la plus complète des niveaux de ${ }^{193,195} \mathrm{Au}$ et s'accorde avec la partie inférieure de la distribution de haute énergie de Gilbert-Cameron (déduite du modèle statistique et des données de capture neutronique). La méthode d'Ericson révèle d'autres aspects intéressants des isotopes de $\mathrm{Pt}$ et $\mathrm{Hg}$ ( $\mathrm{p}$. ex. dépendance entre la densité des niveaux et la forme des noyaux ou des corrélations de paires, évidence pour des transitions de phase). En conséquence, cette méthode est un outil approprié pour l'orientation des recherches expérimentales aussi bien que théoriques des noyaux de transition.

Abstract. - A statistical analysis of $\mathrm{Au}, \mathrm{Pt}, \mathrm{Hg}$ nuclear levels was performed with Ericson's method. The odd mass gold experimental number of levels distributions are compared to the theoretical distributions corresponding to vibrational (Alaga and Kisslinger-Sorensen) and rotational (Stephens, Meyer-ter-Vehn) models. The Alaga model gives the most complete description of ${ }^{193,195} \mathrm{Au}$ levels and fits the lowest part of Gilbert-Cameron high energy distributions (deduced from the statistical model and neutron capture data). The Ericson's method shows other interesting features of $\mathrm{Pt}$ and $\mathrm{Hg}$ isotopes (i.e. level density dependence on nuclear shape and pairing correlations, evidence for phase transitions). Consequently, this method is a useful tool for guiding experimental as well as theoretical investigations of transitional nuclei.
\end{abstract}

1. Introduction. - Au cours des dernières années un progrès considérable a été accompli, en particulier à Orsay et au CERN, dans la détermination des niveaux nucléaires excités de $\mathrm{Hg}, \mathrm{Au}, \mathrm{Pt}$ et Ir déficients en neutrons, aussi bien près que loin de leur zone de stabilité [1-3].

L'étude détaillée de niveaux de basse et moyenne énergie a montré l'influence d'ágrégats de nucléons (ou de lacunes), des changements de forme ou d'appariement sur leurs propriétés. En particulier les niveaux des isotopes d'or les plus lourds $(199 \geqslant A \geqslant 193)$ sont bien décrits par le couplage de trois lacunes de protons dans un caur sphérique de plomb aux vibrations quadrupolaires de la surface du cœur pair-pair (modèle d'Alaga $[4,5]$ ).

Ce modèle donne actuellement la description la plus complète [6] des nombreuses données expérimentales sur les niveaux d'or 193 et 195 sauf peutêtre pour quelques niveaux de parité négative que l'on

$\left({ }^{*}\right)$ Institut de Physique Nucléaire, 91406 Campus Orsay, France. (**) Laboratoire S.-Rosenblum, CSNSM du CNRS, 91406 Orsay, France.

(***) Institute of Physics, University of Stockholm, Sweden.

(****) Fachbereich Physikalische Chemie, Philipps Universität Marburg (RFA). peut attribuer soit à des vibrations octupolaires ( $\left.3^{-}\right)$ du cœur sphérique couplées à des agrégats de lacunes de protons $\left[(3 \mathrm{~s} 1 / 2)^{-1},(2 \mathrm{~d} 3 / 2)^{-1},(2 \mathrm{~d} 5 / 2)^{-1}\right][7]$, soit au couplage d'un proton [1h 9/2] ou d'une lacune de protons [1h 11/2] au mouvement de rotation d'un cœur asymétrique [8-10]. Cependant, tous les calculs actuellement existant sur ce dernier modèle admettent un cour rigide, contrairement aux résultats expérimentaux $[2,11]$ et théoriques [17] concernant les noyaux pair-pair voisins $(\mathrm{Pt}, \mathrm{Hg}$ ).

Dans les études détaillées des noyaux impairs il y a souvent des difficultés considérables à établir une correspondance biunivoque entre les niveaux théoriques et expérimentaux, parfois même à basse énergie. Une confrontation globale entre eux s'impose donc.

Lorsque l'énergie d'excitation croît, la densité des niveaux augmente et on peut appliquer une méthode statistique pour leur analyse. En fait Ericson a montré [12] qu'il est possible d'appliquer de telles méthodes aux distributions (du nombre de niveaux) de basse énergie, même à partir d'une dizaine de niveaux. Dans ce travail, Ericson a démontré, en plus, que ces distributions vérifient la loi suivante : $\log N=\left(E-E_{0}\right) / T$ où $N \equiv N(E)$ est le nombre de 
niveaux d'énergie d'excitation inférieure à $E, E_{0}$ est le seuil de la distribution de l'énergie, $N\left(E_{0}\right)=1, T$ est la température nucléaire moyenne (constante) dans l'intervalle énergétique $\left(E_{0}, E_{\mathrm{m}}\right)$ considéré (avec $E_{\mathrm{m}}<S_{\mathrm{n}}=$ énergie de séparation des neutrons).

Cette loi de distribution a été appliquée aux niveaux des isotopes d'or 193-199 il y a quelques années par Gilbert et Cameron [13]. Cependant, depuis on a augmenté considérablement le nombre de niveaux mis en évidence expérimentalement et plusieurs études théoriques de ces niveaux ont été effectuées dans le cadre des modèles à base sphérique ou déformée. Il est devenu ainsi possible [14] : 1) de comparer valablement les distributions expérimentales entre elles, 2) de confronter celles-ci aux distributions théoriques correspondantes, 3) de rechercher le lien entre ces distributions et celles décrivant les données sur les résonances de capture neutronique radiative.

2. Distributions des niveaux nucléaires excités. Il y a plusieurs types de distributions du nombre de niveaux en fonction de l'énergie d'excitation, $N(E)=f(E)$. Les distributions les plus fréquentes sont celles concernant le nombre de niveaux observés : soit avec une parité donnée $(\pi=+1$ ou -1$)$, soit avec une parité indéterminée $(\pi= \pm 1)$ et un spin $J$ quelconque. Les distributions du nombre (théorique) total de niveaux [13] peuvent se déduire des précédentes en introduisant le facteur $(2 J+1)$ correspondant à la dégénérescence dans le nombre quantique magnétique $M$.

Nous utiliserons les deux types de distributions pour l'analyse statistique des niveaux observés d'or, de platine et de mercure.

2.1 Niveaux D'OR. - De l'analyse des distributions expérimentales des niveaux de parité négative d'or (voir Fig. 1), on tire les conclusions suivantes :

i) Les distributions de ${ }^{191,193,195} \mathrm{Au}$ sont très proches les unes des autres jusqu'à $1,4 \mathrm{MeV}$. Les écarts existant à plus haute énergie proviennent soit de difficultés expérimentales $\left({ }^{191} \mathrm{Au}\right)$ soit de l'énergie totale de désintégration insuffisante pour alimenter ces niveaux de haute énergie.

ii) Les distributions de ${ }^{187,189} \mathrm{Au}$ sont très semblables et présentent un excès systématique de niveaux de basse énergie, par rapport aux autres isotopes d'or.

De l'analyse des distributions théoriques et de leur comparaison aux distributions expérimentales, on tire les conclusions suivantes [14] :

i) Les distributions déduites des modèles vibrationnels à couplage intermédiaire (Alaga [4] et Kisslinger-Sorensen [15]) donnent une description assez bonne des distributions expérimentales jusqu'à 1,6 $\mathrm{MeV}$ pour les trois isotopes d'or considérés $(A=191,193,195)$. Ce fait et l'analogie des distributions expérimentales suggèrent une variation lente

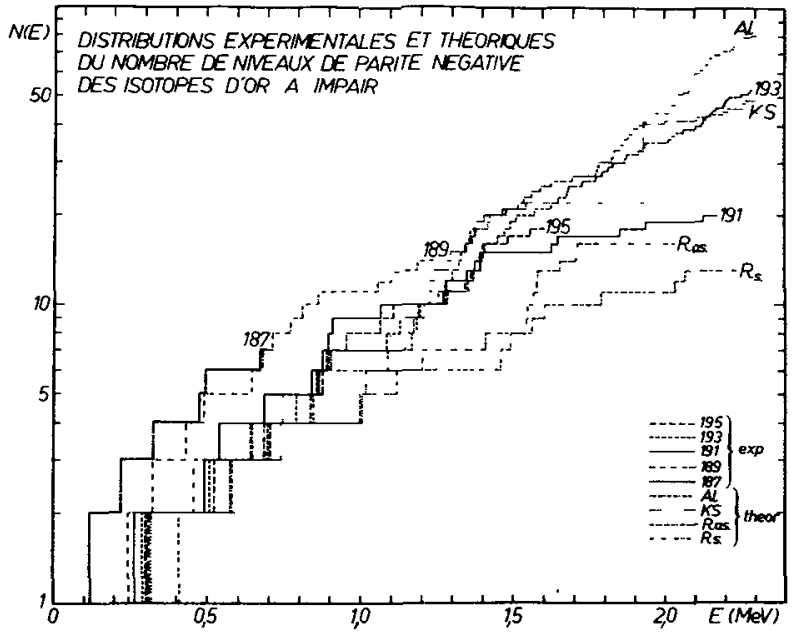

FIG. 1. - Les données utilisées se trouvent référence [14].

des propriétés de ces noyaux de transition qui se comportent donc, en moyenne, comme un ensemble de noyaux sphériques ou peu déformés.

ii) Le modèle à un quasi-proton et trois phonons (KS) ne prédit aucun niveau entre 1,6 et $1,9 \mathrm{MeV}$ contrairement aux faits expérimentaux et aux prévisions du modèle d'Alaga. Ceci démontre l'influence prépondérante des configurations à trois quasi-protons dans ce domaine spectral. L'équivalence entre ces deux modèles pour la description statistique des niveaux de basse énergie ne doit pas faire oublier l'importance de ces configurations dans ces niveaux $[5,6]$.

iii) Les distributions déduites des modèles rotationnels de Stephens [16] et Meyer-ter-Vehn [8] se rapportent à une lacune de protons $(1 \mathrm{~h} \mathrm{11/2)} \mathrm{couplée}$ à un rotor symétrique ou dissymétrique respectivement. Elles dévient considérablement des distributions expérimentales à partir de 1,3 MeV. En admettant la présence de niveaux correspondant à une configuration lh $9 / 2$ parmi les états de basse énergie des isotopes d'or impair, on peut réduire ces écarts. Toutefois, cette hypothèse n'est pas encore entièrement justifiée théoriquement bien que suggérée par les surfaces d'énergie potentielle.

iv) Toutes les distributions théoriques représentées dans cette figure se rapportent à des paramétrisations adaptées à la description des niveaux d'or 195 (aussi bien que l'or 193 dans le cas des modèles d'Alaga et Kisslinger-Sorensen). Les déviations constatées entre ces prévisions et les données expérimentales relatives aux isotopes d'or plus légers résultent soit d'un changement notable de ces paramètres à partir de l'or 191, soit d'une inadaptation des modèles pour leur description.

2. 2 Niveaux De Platine. - Dans la figure 2 sont représentées les distributions du nombre de niveaux observés dans les isotopes de platine pour lesquelles on dispose des données expérimentales suffisantes pour ce type d'analyse. 


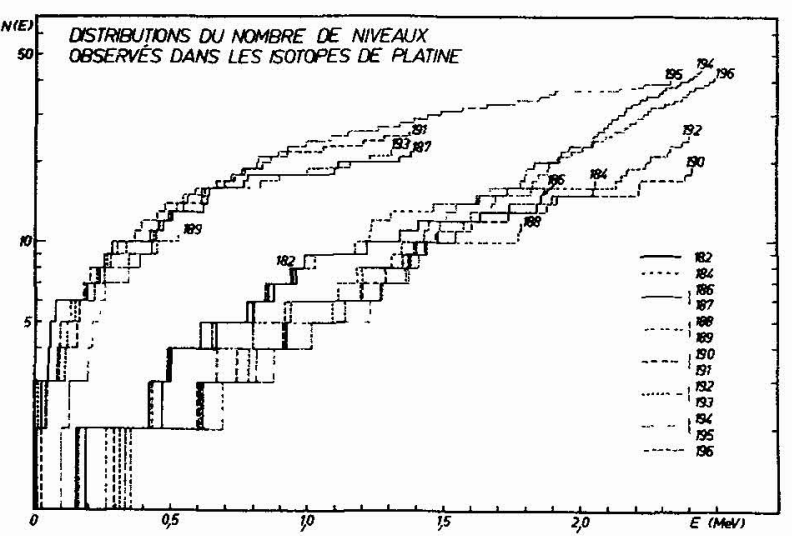

FIG. 2. - Les données utilisées se trouvent dans Nuclear Data Sheets, référence [1] et ce colloque.

La comparaison des distributions expérimentales des isotopes de platine révèle les faits suivants :

i) Les distributions de ${ }^{191,193,195} \mathrm{Pt}$ sont presque identiques entre elles. La partie de basse énergie de la distribution de ${ }^{189} \mathrm{Pt}$ (seule actuellement bien connue et représentée dans la Fig. 2) est presque confondue à celle des précédentes. De même, les distributions de ${ }_{188,190 \ldots 196} \mathrm{Pt}$ se ressemblent.

ii) Les distributions de ${ }^{182,184,186} \mathrm{Pt}$ montrent un excès de niveaux de basse énergie, par rapport aux distributions précédentes, de même que la partie de basse énergie de la distribution du platine 187 .

Jusqu'à présent aucun calcul détaillé des propriétés des niveaux de moyenne énergie des isotopes de platine impairs n'a été effectué dans le cadre des modèles vibrationnels ou rotationnels. De même, ceux dont on dispose sur les isotopes pairs sont relativement peu nombreux [17-19] et concernent exclusivement les niveaux de parité positive.

Toutefois, l'apparition de niveaux $3^{-}, 1^{-}, 5^{-}$, de basse énergie dans les isotopes ${ }^{188 \ldots 196} \mathrm{Pt}$ est révélatrice de vibrations octupolaires ou quadrupolairesoctupolaires et d'effets 'Coriolis caractéristiques de ces noyaux de forme aplatie. Ces effets n'apparaissent pas dans les isotopes de platine pairs plus légers $\left({ }^{182,184,186} \mathrm{Pt}\right)$. Par contre, leur plus grande densité de niveaux de basse énergie suggère une plus grande déformation pour eux. Toutefois, l'abaissement considérable du niveau $0_{2}^{+}$de ces noyaux demeure inexpliqué dans le cadre de nos connaissances actuelles bien que les surfaces d'énergie potentielle montrent la possibilité de deux formes à basse énergie [1].

2.3 NiveauX DE MERCURE. - Dans la figure 3 sont représentées les distributions du nombre de niveaux observés dans les isotopes de mercure pair. De la comparaison de ces distributions on tire les conclusions suivantes :

i) Les distributions de ${ }^{190,198} \mathrm{Hg}$ sont très voisines. Les données actuelles sur les niveaux de ${ }^{192,194,196} \mathrm{Hg}$ suggèrent qu'il doit en être de même pour leurs distributions. La différence entre les distributions de

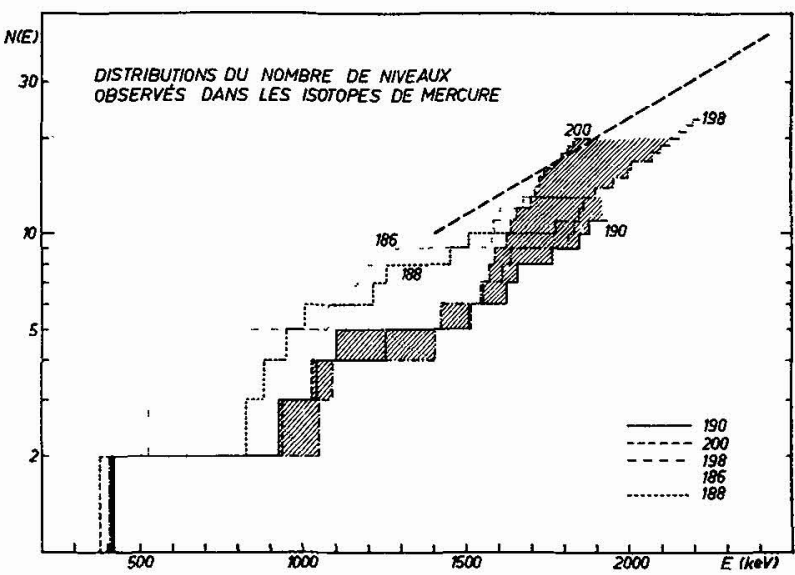

Fig. 3. - Les données incluses dans cette figure sont celles de Nuclear Data Sheets sauf pour le ${ }^{186} \mathrm{Hg}$ (ce colloque) et ${ }^{188,190} \mathrm{Hg}$ obtenues à Isocèle (Orsay) par Foucher, R., LiAnG, C. F. et coll.

${ }^{198} \mathrm{Hg}$ et ${ }^{200} \mathrm{Hg}$ à partir de $1,6 \mathrm{MeV}$ est due au grand nombre de niveaux de bas spin mis en évidence par la capture neutronique dans le mercure 199. Le même défaut doit se retrouver dans les distributions de tous ces isotopes alimentés dans la plupart des cas par la désexcitation d'états à haut spin. Le segment de droite représente la répartition moyenne de la distribution du mercure 200. On n'a pas indiqué les états d'énergie supérieure à $1,8 \mathrm{MeV}$ pour des raisons de simplicité (voir Fig. 4).

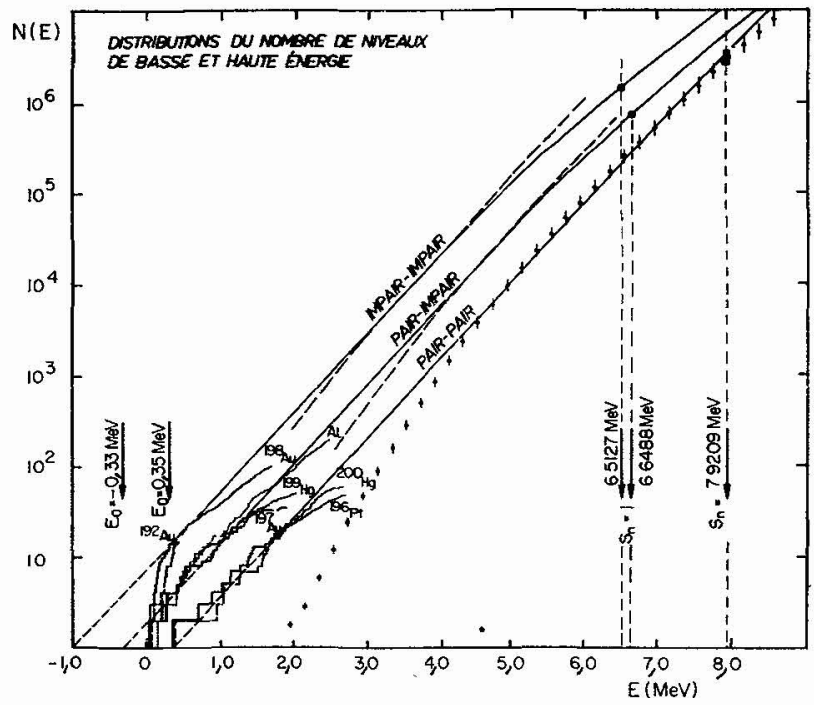

FIG: 4. - Les paramètres des distributions statistiques de GilbertCameron sont donnés dans le tableau III, référence [14].

ii) La distribution du ${ }^{186} \mathrm{Hg}$ semble appartenir à un ensemble différent de celui des distributions des isotopes de mercure plus lourds, comme on l'a constaté dans les isotopes pairs de platine. Il y a néanmoins deux différences entre ces deux sous-ensembles de distributions dans le mercure et le platine : elles sont nettement distinctes dès le premier niveau pour le 
platine (contrairement au mercure); il y a un cas intermédiaire dans le mercure (inexistant dans le platine).

Les calculs effectués pour les isotopes de $\mathrm{Hg}$ pair $[4,19-21]$ les plus lourds montrent l'importance du couplage d'un agrégat de deux lacunes de protons à des vibrations quadrupolaires de surface pour décrire leurs propriétés individuelles et distributions statistiques. En ce qui concerne les isotopes de $\mathrm{Hg}$ légers, cette confrontation n'a pas encore été effectuée mais on constate que les différences observées entre les distributions expérimentales correspondent à des modes de désexcitation différents des niveaux $0_{2}^{+}, 2_{2}^{+}$, $4_{2}^{+}, 6_{1}^{+}$. Ceci suggère l'existence de larges fluctuations d'appariement aussi bien que de forme pour le mercure 186 [22].

2.4 ENERGIES D'APPARIEMENT. - En comparant les distributions de plusieurs nucléides voisins, il est possible de déterminer les énergies d'appariement des neutrons et des protons et de les confronter aux valeurs calculées par Gilbert-Cameron [13]. De cette façon, nous avons obtenu les résultats suivants :

$$
\begin{aligned}
& P\left(N=108 ;{ }^{186} \mathrm{Pt}-{ }^{187} \mathrm{Pt}\right)=0,7 \pm 0,2 ; P_{\text {th }}(N=108)=0,73 ; \\
& \left.\begin{array}{l}
P\left(N=110 ;{ }^{188} \mathrm{Pt}-{ }^{187} \mathrm{Pt}\right)=1,0 \pm 0,2 \\
P\left(N=110 ;{ }^{188} \mathrm{Pt}-{ }^{189} \mathrm{Pt}\right)=0,9 \pm 0,2
\end{array}\right\} P_{\text {th }}(N=110)=0,58 ; \\
& \left.\begin{array}{l}
P\left(N=112 ;{ }^{190} \mathrm{Pt}-{ }^{189} \mathrm{Pt}\right)=1,0 \pm 0,2 \\
P\left(N=112 ;{ }^{190} \mathrm{Pt}-{ }^{191} \mathrm{Pt}\right)=1,1 \pm 0,2
\end{array}\right\} P_{\mathrm{th}}(N=112)=0,86 ;
\end{aligned}
$$

et, de même pour $P(N=114,116$ et 118$)$ ainsi que $P(Z=76,78,80)$ : De même, en adoptant la distribution statistique (ou de haute énergie) de GilbertCameron [13] il a été possible d'accorder les distri- butions de ${ }^{196} \mathrm{Pt},{ }^{198} \mathrm{Au}$ et ${ }^{199} \mathrm{Hg}$ à celles de basse énergie si l'on admet pour ces nucléides l'absence de beaucoup de niveaux expérimentaux et la constance de l'énergie d'appariement dans ce domaine énergétique. Enfin, dans la figure 4, on constate que la distribution d'Alaga s'accorde presque à la partie de basse énergie de la distribution de haute énergie.

3. Conclusions. - Ce travail montre la triple utilité des distributions statistiques d'Ericson pour l'étude des noyaux de transition :

i) Mise en évidence, de façon simple, de sousensembles de noyaux ayant des propriétés de surface analogues (par ex. : isotopes de mercure 190 à 198).

ii) Révélation d'éventuelles lacunes dans les données expérimentales résultant de la comparaison des distributions des nucléides du même type (par ex. : mercure 198-200) ou la suggestion de transitions de phase avec l'apparition de nouveaux modes d'excitation des niveaux (par ex. : imbrication de la forme et de l'appariement dans ${ }^{186} \mathrm{Hg}$ et ${ }^{186} \mathrm{Pt}$ ).

iii) Caractérisation des états (ou particularités) de surface à travers la comparaison entre les distributions théoriques et expérimentales (par ex. : importance des agrégats de trois lacunes de protons pour la description des isotopes d'or impair $A=191$ à 199).

Ce type d'analyse statistique apparaît donc comme un guide précieux pour l'orientation des études théoriques et expérimentales des noyaux de transition.

Remerciements. - Nous remercions vivement nos collègues MM. V. Berg, C. Bourgeois pour nous avoir permis d'utiliser leurs résultats expérimentaux avant leur publication.

\section{Bibliographie}

[1] « Les noyaux de transition, rapport sur l'état actuel des études expérimentales et théoriques " (Colloque Orsay 30 juin2 juillet 1971).

[2] Cailliau, M., Foucher, R. et Husson, J. P., à paraître.

[3] Vieu, Ch. et Dionisio, J. S., Proc. of the Topical Conf. on Problems of vibrational nuclei (Zagreb), ed. G. Alaga, V. Paar et L. Sips, 1974.

[4] AlAGA, G., Rendiconti Scuola Internazionale, Varenna, 40 Corso, 1967, p. 28.

[5] Alaga, G. and PaAR, V., à paraître.

[6] PaAr, V., Vieu, Ch. et Dionisio, J. S., Proc. of the Topical Conf. on Problems of vibrational nuclei (Zagreb), ed. G. Alaga, V. Paar et L. Sips, 1974.

[7] PAAR, V., communication privée.

[8] Meyer-ter-Vehn, J., Stephens, F. et Diamond, R. M., Phys. Rev. Lett. 32 (1974) 1383.

[9] Berg, V., Foucher, R., Hoglund, A., Nucl. Phys. A 244 (1975) 462.

[10] Berg, V., Bourgeois, C. et Foucher, R., J. Physique 36 (1975) 23
[11] Bourgzors, C. et al. (collaboration Isocèle), à paraître.

[12] Ericson, T., Nucl. Phys. 11 (1959) 481.

[13] Gilbert,-A. et Cameron, A. G. W., Can. J. Phys. 43 (1965) 1446.

[14] De Wieclawik, W., Vieu, Ch. et Dionisio, J. S., Proceed. $X X V$ National Conf. on Nuclear Spectroscopy and Structure of the Atomic Nucleus, Leningrad 1975, à paraître.

[15] Kisslinger, L. S. et Sorensen, R. A., Rev. Mod. Phys. 35 (1963) 853.

[16] TృøM, P. O., Maier, M. R., Benson, D., Stephens, F. S. et Diamond, R. M., Nucl. Phys. A 231 (1974) 397.

[17] Kumar, K. et Baranger, M., Nucl. Phys. A 122 (1968) 273.

[18] SCHARFF-Goldhaber, G., CERN 70-30, Genève 1970, vol. 2, 813.

[19] MEYRR-Lévy, M., Thèse Lyon (1973).

[20] Covello, A., Sartoris, G., Nucl. Phys. A 104 (1967) 189.

[21] Alaga, G. et PaAR, V., pre-print (juillet 1974).

[22] Broglia, R. A., Bès, D., Nilsson, B. S., pre-print (1975). 